Meta

Journal des tradlucteurs

Translators' Journal

\title{
Lexicographie bilingue et traduction
}

\section{Brigitte Lépinette}

Volume 35, numéro 3, septembre 1990

La traduction dans le monde hispanolusophone

URI : https://id.erudit.org/iderudit/003468ar

DOI : https://doi.org/10.7202/003468ar

Aller au sommaire du numéro

\section{Éditeur(s)}

Les Presses de l'Université de Montréal

\section{ISSN}

0026-0452 (imprimé)

1492-1421 (numérique)

Découvrir la revue

Citer cet article

Lépinette, B. (1990). Lexicographie bilingue et traduction. Meta, 35(3), 571-581.

https://doi.org/10.7202/003468ar d'utilisation que vous pouvez consulter en ligne.

https://apropos.erudit.org/fr/usagers/politique-dutilisation/ 


\title{
LEXICOGRAPHIE BILINGUE ET TRADUCTION
}

\author{
BRIGITTE LÉPINETTE \\ Universidad de Valencia, Valence, Espagne
}

\begin{abstract}
Dans cet article nous montrons d'abord que 1. la lexicographie bilingue a fait l'objet de travaux théoriques relativement peu nombreux $; 2$. les informations qu'un encodeur peu compétent doit trouver dans un ouvrage lexicographique bilingue pour réaliser correctement l'opération d'encodage sont de nature : a) sémantique, b) syntaxique et c) lexícale (cooccurrences fréquentes du lexème-entrée); 3. ces informations n'apparaissent jamais systématiquement dans un dictionnaire bilingue conventionnel. Nous proposons ensuite quelques articles d'un dictionnaire explicatif et combinatoire bilingue pratique qui présentent les données que nous montrons être nécessaires à cette opération d'encodage. Ce dictionnaire (en cours d'élaboration), dont les entrées seront dans un premier temps constituées par les lexèmes espagnols, équivalences interlinguales des items verbaux de l'inventaire du français fondamental, s'appuiera systématiquement sur une analyse distributionnelle de la langue cible. Il représente une tentative pratique de rénovation de la lexicographie bilingue diversifiée en fonction des besoins des utilisateurs.

In this paper, we show that 1) the bilingual lexicography is a field that has not often been object of research; 2) the information that a non-expert translator must find in a bilingual lexicography work in order to realize properly the codification process, are of three different sorts: a) semantical, b) syntactical and c) lexical (recurrent combinations of each entry); 3) this information does not systematically appear in a conventional bilingual dictionary. We present in this paper, some articles from the B.E.C.D. (bilingual explanatory and combinato$r y$ dictionary), that contain the information we dernonstrate to be necessary in a codification process. This dictionary's entries will first be the Spanish lexemes, linguistic equivalences of the verbal items of the F.F. inventory. The basis of this dictionary will be a distributional analysis of the target-language. So this work will represent a practical essay to renovate the bilingual lexicography in such a way to satisfy the users' needs.
\end{abstract}

0.1 Nous entendrons seulement ici par «traduction» (et ceci d'une manière évidemment arbitraire) l'opération d'«encodage», c'est-à-dire dans notre situation, la transformation en français (langue cible ou $\mathrm{L}^{2}$ ), d'un texte originellement en espagnol (langue source ou $\mathrm{L}^{1}$ ) par un sujet dont la compétence en langue cible (français) n'est pas celle d'un locuteur francophone. Si cette situation n'est pas la plus courante, la plupart des traducteurs produisant des textes dans leur langue maternelle, elle n'est cependant pas rare, loin de là. 0.2 Les travaux concernant la lexicographie bilingue en général et en particulier la lexicographie français-espagnol (voir bibliographie) ne sont pas très nombreux, surtout si nous les comparons aux publications qui se sont occupées de la lexicographie monolingue ces trente dernières années. Cependant les études récentes sur ce sujet (Zgusta, 1984; Snell-Horby, 1986; Wexler, 1987) montrent, dans des perspectives différentes, la nécessité pour les lexicographes bilingues de se démarquer de techniques qui ont leur origine dans la lexicographie monolingue ${ }^{1}$ et aussi de modifier le modèle unique du dictionnaire bilingue conventionnel en fonction de besoins d'utilisateurs dont le profil sera préalablement déterminé2 
0.3 Certains travaux actuels mettent en évidence la possibilité d'application de leurs analyses à une lexicographie bilingue différente de la conventionnelle. Nous signalerons deux directions que nous rencontrerons à nouveau ultérieurement dans notre développement. Ce sont les travaux d'Elia (1984) qui analyse contrastivement, dans le cadre du Lexique-grammaire élaboré par le L.A.D.L. ${ }^{3}$, un ensemble de vocables français et italiens et ceux de Lépinette (1988a, 1988b), qui appliquent à la lexicographie bilingue les principes établis par Mel'čuk (1984) dans son ouvrage monolingue : Dictionnaire explicatif et combinatoire du français contemporain. Ces travaux cependant se situent dans un domaine qui est celui de la recherche linguistique théorique et dans un cadre qui conduira à la création d'un volume d'information dont il faudrait tester l'intérêt s'agissant de la traduction: les besoins d'un traducteur sont d'ordre pratique. Les résultats d'analyses théoriques exhaustives doivent peut-être être simplifiés en fonction de ce but et en tout cas ils doivent être organisés en fonction de celui-ci.

0.4 Certaines réalisations lexicographiques pratiques, bien que monolingues, sont intéressantes pour nous. L'une d'elles constitue un exemple de description du comportement syntaxique des termes vedettes et à la fois de la présentation de leur cooccurrence lexicale restreinte, ces deux aspects étant indispensables pour un encodage correct de la part d'un sujet peu compétent, nous le verrons un peu plus longuement infra. Il s'agit du B.B.I. (Benson et al., 1986). Cependant, disons dès maintenant que si ce dictionnaire décrit bien la relation syntaxique entre le terme vedette et les éléments qu'il régit dans les grammatical collocations et s'il détermine effectivement les cooccurrences fréquentes, lexical collocations, il ne tient pas compte des propriétés sémantiques des items lexicaux décrits, qui doivent d'une manière indispensable faire l'objet d'une information dans un dictionnaire bilingue comme celui que nous envisageons.

1. Nous partons du postulat suivant: pour la réalisation correcte de l'opération d'encodage, le sujet peu compétent en langue cible doit disposer d'un instrument qui lui offre les données nécessaires pour:

1) effectuer un choix correct de l'équivalence sémantique en $L^{2}$ de l'item visé en $L^{1}$;

2) insérer correctement dans la phrase qu'il encode chacun des éléments lexicaux qu'il veut y intégrer;

3) associer chacun d'eux dans une séquence qui serait celle qu'attend un locuteur compétent. Les données nécessaires pour un encodage correct de la part d'un sujet peu compétent sont donc de nature à la fois sémantique et syntaxique et lexicale (inclusion de la cooccurrence fréquente).

Dans une communication présentée à la réunion annuelle de l'Association espagnole de linguistique appliquée (Lépinette, 1988c), nous avons montré que ces trois tâches du dictionnaire bilingue, parce qu'il est essentiellement héritier d'un système d'équivalences interlinguales semblables à des étiquettes ${ }^{4}$, sont remplies de façon lacunaire et asystématique par le recours à toute une gamme de procédés pour lesquels la normalisation est loin d'être la règle. Ces ouvrages voulant servir à tout, ils sont souvent inadéquats pour leurs utilisateurs, car ce qui devrait être le principe de base de la lexicographie bilingue - une organisation en fonction de la réalisation de l'encodage en langue cible ou de celle du décodage de cette même langue — n'est jamais envisagé : les recueils français-espagnol actuels ne distinguent ni théoriquement (dans leurs prologues explicitant leurs options dictionnairiques) ni pratiquement (dans l'élaboration de leurs articles) ce qui est utile dans une opération de décodage de ce qui sert dans l'opération d'encodage. Cette dernière affirmation a été démontrée dans les commentaires des articles banqueroute et loger extraits du Diccionario moderno francés-español, español-francés (GarcíaPelayo, 1987) que nous effectuons dans la communication citée supra. 
Signalons en outre que quand des données de nature sémantique, syntaxique ou lexicale figurent dans un dictionnaire bilingue, elles sont choisies intuitivement par le lexicographe sans que celui-ci se soit appuyé, au moment de la sélection de ces dernières, sur une méthode d'analyse linguistique déterminée, aux principes clairement énoncés et en cohérence avec les objectifs qu'il aurait dû se marquer. Nous renvoyons également à ce sujet à la communication déjà citée.

1.1 Nécessité de l'information sémantique sur l'item lexical en langue cible, en entrée dans un dictionnaire d'encodage

L'analyse de l'insuffisance ou de l'inadéquation de l'information fournie par les dictionnaires bilingues conventionnels, que nous avons effectuée dans la communication signalée, nous a permis de vérifier que l'encodeur doit trouver sous l'entrée, en plus d'une équivalence linguistique en langue cibles, tout d'abord une information sur le sémantisme de l'item lexical $\mathrm{X}^{2}$ de $\mathrm{L}^{2}$ présenté comme correspondant $\mathrm{X}^{1}$ de $\mathrm{L}^{1}$. S'il existe ce que l'on pourrait appeler un isosémantisme entre $X^{1}$ et $X^{2}$, c'est-à-dire une équivalence sémantique parfaite entre $X^{1}$ et $X^{2}$ (ce qui est courant seulement quand il s'agit d'unités lexicales monosémiques, la plupart des cas appartenant à des lexiques techniques et scientifiques), la question ne se pose évidemment pas; mais si au contraire - cas fréquent $-\mathrm{X}^{1}$ en entrée est suivi dans le dictionnaire conventionnel de $\mathrm{X}^{2}, \mathrm{Y}^{2}, \mathrm{Z}^{2}$, l'encodeur doit trouver des critères à l'intérieur de l'article pour pouvoir, au moment de l'encodage, choisir comme équivalence linguistique de $X^{1}$ entre $X^{2}, Y^{2}$ et $Z^{2}$. Les items $\mathrm{X}^{2}, \mathrm{Y}^{2}, \mathrm{Z}^{2}$ doivent faire l'objet d'une information sémantique en $\mathrm{L}^{1}$. Ainsi frontera, terme choisi au hasard dans le dictionnaire bilingue conventionnel qui nous fournit nos exemples, y est présenté comme équivalent de frontière mais aussi de limite et de ligne sans qu'un encodeur peu compétent en français ait de données objectives pour choisir l'un ou l'autre terme. Un hispanophone s'appuiera pour effectuer son choix sur des similitudes morphologiques avec des items de sa propre langue, déduisant de ces similitudes morphologiques des équivalences sémantiques. Mais évidemment ceci constitue une pratique peu sûre du point de vue des résultats.

Dans le cas de notre dictionnaire d'encodage, une solution possible mais que nous avons écartée aurait été un véritable système définitionnel. C'est la solution que nous avons préconisée dans Lépinette (1988a et 1989). Cette dernière est satisfaisante du point de vue théorique concernant le contraste des langues. Dans une perspective pratique cependant, il nous semble nécessaire d'adopter un système de caractérisation sémantique plus facilement normalisable et plus économique qu'une définition. C'est pourquoi, dans notre propre ouvrage, nous essayons de nous limiter à une seule équivalence intralinguale - en $\mathrm{L}^{1}$, langue de l'encodeur - chaque fois que cela est possible.

Cette solution paraît adéquate si nous la complétons à l'aide d'un système simplifié d'analyse sémantico-syntaxique qui met en évidence les propriétés distributionnelles des lexèmes traités de $L^{2}$ (propriétés qui sont précisément celles sur lesquelles nous nous appuyons pour la séparation d'un item lexical donné en divers lexèmes). Ce sont celles-ci qui, unies à des notions proprement syntaxiques que nous énumérerons, nous autorisent à séparer par ex. se moquer 1: se moquer de Nhum. et se moquer 2: se moquer de Nabstr. (Max se moque de Léo/Max se moque du conseil) dans notre article se moquer, qui correspondent à deux lexèmes (reirse de/no importarle a alguien algo). Ce système est constitué, du point de vue sémantique, par les propriétés des substantifs en position de sujet du verbe et de complément direct: Nhum/N-Hum/Nnr (c.-à-d. verbe à l'infinitif ou proposition), Nconcr (Nconcret: énumérable/non énumérable)/Nabst. Notons que nous suivons en cela les principes qui ont présidé à l'établissement des analyses - souvent non menées jusqu'à leurs dernières conséquences - d'élaboration des articles d'un dictionnaire monolingue : le Dictionnaire du français contemporain (Dubois et al., 1966). 
Ce double système de caractérisation sémantique : équivalence intralinguale en $\mathrm{L}^{1}$ + schéma de phrase dans laquelle est intégré le lexème équivalence interlinguale en $\mathrm{L}^{2}$ avec les restrictions d'emploi qu'il impose, nous conduit:

a) d'un point de vue pratique, à la mise en place d'un système d'équivalences interlinguales, efficace et clair. Plaindre 1 sera ainsi mis en relation avec compadecer, et (se) plaindre 2 avec quejarse défini en entrée pour les séparer de ses homonymes-homographes: expresar su sufrimiento (cf. le macro-article plaindre que nous présentons infra);

b) d'un point de vue théorique et pour chaque lexème en $\mathrm{L}^{2}$, à une analyse sémantique systématique s'appuyant sur des critères objectifs ${ }^{6}$.

1.2 Nécessité de l'information syntaxique sur l'item lexical de la langue cible en entrée dans le dictionnaire

Pour encoder correctement, l'utilisateur du dictionnaire bilingue doit connaître les règles d'insertion dans la phrase, du lexème visé avec les autres éléments qui composent celle-ci, pour éviter de produire des phrases inacceptables. Le dictionnaire doit donc être non seulement sémantique, mais syntaxique.

Dans l'optique qui est la nôtre et qui consiste à «marier» sémantique et syntaxe, c'est dans les études du lexique-grammaire du L.A.D.L. (Gross, Guillet, 1984) que nous pouvons trouver le modèle le plus satisfaisant, mais qui sera évidemment à simplifier dans une optique pratique. En effet le lexique-grammaire présente des analyses exhaustives de type distributionnel et transformationnel et fournit pour chaque item lexical des données syntaxiques complètes. Ces données, en principe destinées à «nourrir» des machines pour une exploitation informatique, représentent un ensemble non directement et totalement utilisable dans un ouvrage lexicographique pratique, à notre avis précisément pour des raisons de volume.

Nous indiquons dans nos articles, le(s) type(s) de construction(s) dans le(s)quel(s) s'insère le lexème vedette traité:

- constructions simples: par ex.: $\mathrm{No}+\mathrm{V}+$ à $\mathrm{N}^{1}+$ de $\mathrm{N}^{2}$ (Max parle à Léa de la pluie);

- constructions complexes (complétives) : par ex.: No V N1 de $\mathrm{V}^{1}$ inf. (Max envie Léa de faire cela).

La présence obligatoire des compléments direct $\mathrm{N}^{1}$ et indirect $\mathrm{N}^{2}$ est toujours prise en compte. La nature des relations prépositionnelles est indiquée. Les modes des propositions complétives figurent.

1.3 Nécessité de l'information sur la cooccurrence lexicale restreinte de l'item lexical en langue cible en entrée dans le dictionnaire

Le modèle le plus adéquat, en ce qui concerne la présentation et la classification de la cooccurrence lexicale restreinte pour un lexème donné, est celui que nous trouvons dans le D.E.C. (Mel'čuk et al., 1984). Ce dictionnaire définit la cooccurrence lexicale restreinte de la manière suivante (p. 5): «il existe cooccurrence lexicale restreinte si un lexème $A$ signifiant ' $A$ ' et un lexème $B$ signifiant ' $B$ ' ne peuvent pas se combiner pour exprimer le sens composé ' $A+B$ ', cela n'étant pas interdit par la syntaxe». Cet ouvrage présente ainsi la combinatoire lexicale du lexème en entrée en la décrivant et la classant systématiquement selon les fonctions lexicales (FL) déterminées (p. 6): «Une FL est un sens assez abstrait (de type 'très', 'commencer', 'capable de', 'réaliser') ou un certain rôle sémantico-syntaxique tel que son expression linguistique dépend du lexème auquel cette FL s'applique. Ce lexème est dit argument de la FL (...). Nous avons trouvé $60 \mathrm{FL}$ (...). Pour chaque lexème répertorié, le D.E.C. donne toutes les valeurs de toutes les FL dont ce lexème peut être l'argument.» L'intérêt du travail de Mel'čuk apparaît clairement, 
si on le compare aux présentations d'expressions figées ou semi-figées que la lexicographie conventionnelle nous offre: celles-ci sont toujours en vrac sans autre ordre que l'alphabétique. Au contraire, il existe dans le D.E.C. une approche méthodique et extrêmement cohérente de la question de la description et de l'ordonnancement de ces expressions.

Cependant dans la pratique lexicographique bilingue, nous pensons, d'une part, que la spécification du rôle sémantico-syntaxique (c.-à-d. du rapport avec le lexème vedette) n'est pas indispensable. Nous optons donc pour classer ces cooccurrences lexicales uniquement selon leur catégorie syntaxique et sans préciser le rapport syntaxico-sémantique de ces cooccurrents fréquents avec le lexème vedette comme le faisait Mel'čuk, puisque, entre autres raisons, la présentation en premier lieu de l'équivalence de ceux-ci en $\mathbf{L}^{1}$ rend superflue une analyse abstraite de ce rôle par rapport au lexème vedette.

2. Nous proposons ici quelques articles de dictionnaire bilingue apportant les renseignements que nous avons montré être nécessaires pour l'encodage. Notre but étant de fournir à des hispanophones peu compétents en français des équivalences espagnoles des lexèmes français correspondant aux items lexicaux sélectionnés dans le Dictionnaire fondamental de la langue française (Gougenheim, 1958), nous présentons celles-ci dans une suite d'équivalences espagnol-français. Dans une optique d'encodage, ce dictionnaire doit en effet comporter, dans une première partie, un index alphabétique avec les entrées en espagnol $\left(\mathrm{L}^{1}\right)$ qui renvoient aux articles dont l'entrée est française $\left(\mathrm{L}^{2}\right)$, la seconde partie étant constituée par les articles eux-mêmes établis selon les principes énoncés supra et fournissant donc le comportement syntaxique et la cooccurrence lexicale fréquente du verbe en entrée et aussi les dérivés qui se rattachent sémantiquement à ce dernier. Pour les entrées espagnoles polysémiques, un système de phrases en espagnol (cf. exemple de l'index: coger) permet le choix de l'item français correspondant. Cette double recherche correspond aux deux moments de l'opération d'encodage: 1. la recherche de l'équivalence en $L^{2}$ et 2 . la manière d'intégrer correctement celle-ci dans une phrase.

Les articles eux-mêmes établis selon les principes énoncés supra fournissent donc d'abord le comportement syntaxique du lexème vedette et les dérivés qui s'y rattachent sémantiquement (produits d'opérations de transformations), ensuite la cooccurrence lexicale fréquente.

Comme cela doit se dégager de cette description de l'organisation que nous venons de faire, celle-ci représente le résultat d'une tentative pour combiner deux finalités différentes, la première d'ordre thérique et la seconde d'ordre pratique, qui sont les suivantes:

a) présenter une analyse linguistique $\left(\mathrm{de}_{\mathrm{L}}\right)$ systématiquement organisée selon des principes clairement énoncés: dégroupement sur des bases sémanticosyntaxiques des lexèmes, description de leur fonctionnement syntaxique, présentation des dérivés qui s'y rattachent sémantiquement, présentation de la cooccurrence lexicale fréquente;

b) permettre la lecture et mettre à profit la description de données décrites systématiquement pour un lexème en $L^{2}$ en vue de la réalisation d'un encodage correct.

En ce qui concerne notre choix d'un corpus de verbes dont la sélection a été opérée avec les critères du français fondamental, nous dirons que :

a) ce corpus, d'un point de vue strictement pratique, représente un objet d'analyse de taille encore maniable, compte tenu de la dimension des articles que nous y introduisons. En tout état de cause, nous sommes consciente que cette première 
partie devrait être immédiatement complétée par une analyse des substantifs du français fondamental pour former un ensemble plus complet et systématique. Cependant, plutôt que l'importance numérique de l'inventaire des items traités, ce qui nous intéresse ici au premier chef, c'est la cohérence de l'analyse (selon les critères théoriques que nous avons déterminés) et l'adéquation de celle-ci aux besoins d'encodeurs peu compétents;

b) ce corpus représente à notre connaissance la première analyse sémantique reposant sur des bases théoriques clairement énoncées du contraste entre ces items lexicaux de $\mathrm{L}^{2}$ (et leur cooccurrence) et leurs correspondants en $\mathrm{L}^{1}$. Plusieurs inventaires bilingues du F.F. ont été réalisés en Espagne ${ }^{7}$ mais ils se situent tous dans la lignée des dictionnaires bilingues conventionnels et n'ont pas mis à profit, en ce qui concerne les techniques lexicographiques, les apports d'analyses linguistiques récentes.

2.1 Nous présenterons trois articles de notre dictionnaire bilingue correspondant aux verbes arranger et attraper, et se plaindre ${ }^{8}$. Un des trois verbes est un opérateur. Les deux premiers articles seront donc plus économiques que le dernier. Comme nous l'avons montré plus haut, chaque analyse doit être précédée par l'intégration dans l'index de tous les lexèmes qui y sont traités. Lorsque ces lexèmes sont les entrées des articles, ils apparaissent accompagnés d'une équivalence intralinguale. Lorsqu'ils figurent seulement à titre de cooccurrents à l'intérieur de l'article, ils apparaissent alors avec un seul renvoi à l'entrée sous laquelle ils sont classés et analysés. L'index que nous présenterons ici est évidemment partiel, il est uniquement le produit de l'analyse des trois verbes français traités. Nous le faisons figurer fragmenté en trois parties correspondant aux trois articles concernés.

\subsection{Index}

\subsubsection{Indice de Arranger}

Arreglar: Cf. arranger 1 ((re)poner en un estado satisfactorio algo deteriorado, reparar). Arreglar: Cf. arranger 2 (resolver una dificultad, un asunto difícil, una situación difícil, unas relaciones dificiles entre personas).

Arreglarselas: Cf. arranger 3 (actuar de la manera más conveniente posible).

Arreglo: Cf. arranger 1

Arreglo: Cf. arranger 2

Conciliador: Cf. arranger 2

Convenir: Cf. arranger 4 (ser satisfactorio).

\subsubsection{Indice de Attraper}

Alcanzar: Cf. attraper 1 (alcanzar un objeto en movimiento)

Atrapar: Cf. attraper 2 (alcanzar un ser animado que escapa)

Coger: Cf. attraper 1 (alcanzar un objeto en movimiento)

Coger: Cf. attraper 2 (alcanzar un ser animado que escapa)

Engañar: Cf. attraper 3 (hacer creer que algo es verdad/bueno/justo, etc., cuando no lo es)

Engaño: Cf. attraper 3

Reprimenda: Cf. attraper 4

Reñir: Cf. attraper 4 (regañar, reprender)

Trampa: Cf. attraper 3 


\subsubsection{Indice de Se plaindre}

Compadecer (se) Cf. plaindre 1 (sentir compasión)

Demandar: Cf. (se) plaindre 4

Denunciar: Cf. (se) plaindre 4 (denunciar)

Lastimero: $\mathrm{Cf}$. (se) plaindre 2

Lastimosamente: Cf. (se) plaindre 2

Quedamente: Cf. (se) plaindre 2

Queja: Cf. (se) plaindre $2+3$

Quejarse: Cf. (se) plaindre 2 (expresar su sufrimiento)

Quejarse: Cf. (se) plaindre 3 (expresar su descontento)

Quejica: Cf. (se) plaindre 2

Quejicoso: Cf. (se) plaindre 2

Quejido: Cf. (se) plaindre 2

Quejoso: Cf. (se) plaindre 3

Quejumbroso: Cf. (se) plaindre 2

Querella: 1 - Cf. (se) plaindre 4

Silenciosamente: $\mathrm{Cf}$. (se) plaindre 2

\subsubsection{Article Arranger}

1 - Arranger ((re)poner en un estado satisfactorio algo deteriorado, reparar $)=$ arreglar Constr. : Max arregla la puerta, el motor, el jersey (Nhum $+\mathrm{V}+\mathrm{Nconcr})=\mathrm{Max}$ arrange la porte, le moteur, le lainage (Nhum $+\mathrm{V}+\mathrm{Nconcr})$. Der. Un arreglo, una reparación $=$ un arrangement.

Cooc. lex.: V. Hacer un arreglo (cuidado): faire un arrangement (rapide). Adj. Un arreglo costoso: un arrangement coûteux.

Un arreglo rápido, chapucero: un arrangement bâclé. Un arreglo cuidado: un arrangement soigné. Adv. (Arreglado, rehecho) a conciencia / (arranger) soigneusement.

2 - Arranger (resolver una dificultad, un asunto difícil, una situación dificil, unas relaciones dificiles entre personas) $=$ Arreglar .

Constr. : a - Max arregla un asunto complicado (Nhum + V + Nabstr)/Max arrange une affaire compliquée (Nhum $+\mathrm{V}+\mathrm{Nabstr)}$ (transf.: un asunto complicado se arregla [gracias a Max] (Nabstr + se V + [prep Nhum])/ une affaire compliquée s'arrange [grâce à Max $](\mathrm{Nabstr}+$ se $\mathrm{V}[+$ prep Nhum] $)$.

$\mathrm{b}-$ Max se arregla con Leo en el precio de la casa (Nhum $+\mathrm{V}+$ con Nhum en + Nabstr)/Max s'arrange avec Léo pour le prix de la maison (Nhum $+\mathrm{V}+$ avec Nhum + pour Nabstr).

- Max et Leo se arreglan en el precio de la casa (Nhum + se V + en Nabstr)/Max et Léo s'arrangent pour le prix de la maison (Nhum + se $\mathrm{V}+$ pour Nabstr).

- Max et Leo se arreglan amistosamente (Nhum $+\mathrm{se} \mathrm{V}+\mathrm{adv}$ )/Max et Léo s'arrangent à l'amiable (Nhum + se V + adv).

Der. N. Un arreglo/un arrangement. Adj. (Una persona) conciliador(a)/(une personne) arrangeant(e).

Cooc. lex. : Adj. (Un arreglo) diplomático/(un arrangement) diplomatique. (Un arreglo) dificil/ (un arrangement) difficile. (Un arreglo) amistoso / un arrangement à l'amiable.

3 - S'arranger (actuar de la manera más conveniente posible) = arreglarselas

Constr.: Max se las arregla [para sobrevivir - con una sola mano] (Nhum + V [= para 
Vinf]/con N)/Max s'arrange [pour survivre]/avec une seule main (Nhum $+\mathrm{V}$ [pour Vinf / avec N]).

4 - Arranger (ser satisfactorio) $=$ convenir, venir bien

Constr.: Esta situación/esta mesa/que llegues pronto conviene a Max $(\mathrm{Nnr}+\mathrm{V}+\mathrm{a}$ Nhum)/cette situation/cette table/que tu arrives tôt arrange $\mathrm{Max}(\mathrm{Nnr}+\mathrm{V}+\mathrm{Nhum})$.

\subsubsection{Article Attraper}

1 - Attraper (alcanzar un objeto en movimiento $=$ Max attrape le ballon, le train) $=$ coger, alcanzar.

Constr.: Max alcanza-coge la pelotalel tren que iba a arrancar (Nhum $+\mathrm{V}+$ Nconcr)/Max attrape le ballon/le train (Nhum + V + Nconcr).

Cooc. lex.: Adv.: al vuelo/au vol (coger al vuelo/attraper au vol).

2 - Attraper (alcanzar y detener un ser animado que escapa-puede escapar = Max attrape le voleur $)=$ coger, atrapar .

Constr.: El gato coge el ratón (Nanim $+\mathrm{V}+\mathrm{Nanim})$ / le chat attrape la souris (Nanim + $\mathrm{V}+$ Nanim). Max coge mariposas/moscas (Nhum $+\mathrm{V}+\mathrm{Nanim}) / \mathrm{Max}$ attrape des papillons, des mouches. Max coge al ladrón (Nhum $+\mathrm{V}+\mathrm{Nhum}$ )/Max attrape le voleur (Nhum $+\mathrm{V}+$ Nhum).

Cooc. lex.: Adv.: Coger por sorpresa: attraper par surprise.

3 - Attraper (Max hace creer a Leo que algo es verdad/bueno / justo, etc., cuando no lo es y Leo cae en la trampa) = engañar.

Constr.: Max engaña a María [siempre / con su aspecto inocente] (Nhum $+\mathrm{V}+\mathrm{Nhum}$ ) $=$ Max attrape Marie [chaque fois / avec son air innocent] (Nhum $+\mathrm{V}+\mathrm{Nhum})$.

Der. : un engaño, una trampa: une attrape, un attrape-nigaud.

4 - Attraper (regañar, reprender) $=$ reñir.

Constr.: Max riñe a María $($ Nhum $+\mathrm{V}+$ Nhum $)=$ Max attrape Marie $($ Nhum $+\mathrm{V}+$ Nhum).

Der.: Una reprimenda: une attrapade.

5 - Attraper (contraer una enfermedad $)=$ coger .

Constr.: Max coge un catarro (Nhum $+\mathrm{V}+\mathrm{N}$ de maladie) /Max attrape un rhume (Nhum $+\mathrm{V}+\mathrm{N}$ de maladie ).

\subsubsection{Article Plaindre, se plaindre}

1 - Plaindre (sentir compasión, lástima = éprouver un sentiment de compassion, de pitié) $=$ compadecerse .

Constr.: a - Max se compadece de Leo (Nhum $+\mathrm{V}+$ de Nhum)/Max plaint Léo (Nhum + V + Nhum). b - Max se compadece de Leo por / a causa de su enfermedad (Nhum $+\mathrm{V}+$ de Nhum por Nabst) / Max plaint Léo de sa maladie (Nhum $+\mathrm{V}+\mathrm{Nhum}$ de Nabst). $\mathrm{c}-$ Max se compadece de Leo porque está enfermo ( $\mathrm{Nhum}+\mathrm{V}+\mathrm{de}$ Nhum porque $\mathrm{P}$ ind.) / Max plaint Léo d'être malade / de ce qu'il n'a/ait pas réussi (Nhum $+\mathrm{V}+$ Nhum de + Vinf./ de ce que P ind. / subj.).

Cooc. lex. : Adv. : sinceramente/sincèrement.

2 - Plaindre (expresar un / su sufrimiento / exprimer de la / sa souffrance $)=q u e j a r s e$.

Constr.: a - Max se queja de su enfermedad - por el mal recibimiento (Nhum + V + de/por $\mathrm{N}$ )/Max se plaint de sa maladie - du mauvais accueil (Nhum $+\mathrm{V}+\mathrm{de} \mathrm{N}) . \mathrm{b}-$ Max se queja de su enfermedad - por el mal recibimiento a Leo (Nhum $+\mathrm{V}+\mathrm{de} /$ por $\mathrm{N}$ 
+ a Nhum)/Max se plaint de sa maladie - du mauvais accueil à Léo (Nhum $+\mathrm{V}+\mathrm{de} \mathrm{N}$ + a Nhum). $\mathrm{c}-$ Max se queja porque está enfermo (Nhum $+\mathrm{V}+$ porque $\mathrm{P}$ ind.)/Max se plaint de ce qu'il est malade (Nhum $+\mathrm{V}+$ de ce que $\mathrm{P}$ ind.).

Der.: Queja/Plainte: La(s) queja(s) del enfermo/la (les) plainte(s) du malade. a - un quejido [el quejido del enfermo]/un cri de plainte [le cri de plainte chaque fois qu'on déplaçait le malade]. $\mathbf{b}$ - (una persona) quejumbrosa/une personne plaintive; alguien que se queja exageradamente de males físicos sin importancia $=$ un quejica/(personne qui se plaint exagérément de maux physiques sans importance) [un douillet]. $\mathrm{c}$ - (un sonido - grito) lastimero/ (un son - cri) plaintif.

Cooc. lex.: V.: (quejarse)/Émettre, proférer des plaintes (Nhum. $+\mathrm{V}+-+$ de Nhum). Provocar - causar/Provoquer - causer les plaintes de $(\mathrm{N}+\mathrm{V}+-+$ de Nhum). Disminuir - calmarse/Se calmer, s'apaiser (- + V) [Las quejas del enfermo disminuyeron con el efecto del sedante/les plaintes du malade s'apaisèrent sous l'effet du calmant]. Adj.: Unas quejas lastimeras/une (des) plainte(s) émouvante(s), - qui fendent l'âme, qui crèvent le cœur. Unas quejas débiles / une (des) plainte(s) faible(s).

Unas quejas ahogadas/une(des) plainte(s) retenue(s), étouffée(s). Adv.: (quejarse) quedamente /(se plaindre) doucement. (quejarse) silenciosamente/(se plaindre) silencieusement. (quejarse) lastimosamente / (se plaindre) pitoyablement.

3 - Plaindre (expresar el descontento/ exprimer du / son mécontentement) $=$ quejarse .

Constr. : a - Max se queja de Leo-de la injusticia (Nhum + V + de N)/Max se plaint de Léo - de l'injustice (Nhum $+\mathrm{V}+$ de $\mathrm{N}) . \mathrm{b}-$ Max se queja de Leo-de la injusticia a Ida (Nhum $+\mathrm{V}+$ de $\mathrm{N}+\mathrm{Nhum}) / \mathrm{Max}$ se plaint de Léo - de l'injustice à Ida (Nhum + $\mathrm{V}+$ de $\mathrm{N}$ à Nhum). $\mathrm{c}-$ Max se queja de quelporque ha sufrido una injusticia (Nhum + $\mathrm{V}+$ porque $\mathrm{P}$ ind.)/Max se plaint de ce qu'il a subi une injustice (Nhum $+\mathrm{V}+$ de ce que $P$ ind.).

Der.: Queja /Plainte: (persona) quejosa /(personne) qui a à se plaindre lestoy quejoso de tu comportamiento/j'ai à me plaindre de ton comportement].

Cooc. lex. : V. Lanzar, proferir quejas / proférer des plaintes (Nhum $+\mathrm{V}+-$ ) prorrumpir en, deshacerse en quejas / proférer des plaintes bruyantes (ou continuelles) (Nhum $+\mathrm{V}+-$ ). Atender las quejas de Max (Nhum $+\mathrm{V}+-$ de Nhum)/recevoir, prendre note de la(des) plainte(s) de Max (Nhum + V +-de Nhum). Provocar, causar/provoquer, causer (N+V +-) [el estado de las carreteras provoca quejas/l'état des routes provoque des plaintes]. Multiplicarse/se multiplier $(-+\mathrm{V})$ [Las quejas se multiplican/les plaintes se multiplient]. Adj .: Una(s) quejas(s) encendida(s)/une(des) plainte(s) véhémente(s). Una(s) quejas(s) enérgica(s)/une(des) plainte(s) énergique(s). Una(s) queja(s) tímida(s)/ une(des) plainte(s) timide(s). Una(s) queja(s) amarga(s)/une(des) plainte(s) amère(s). Una(s) queja(s) repetida(s) frecuente(s)/une(des) plainte(s) répétée(s), réitérée(s), fréquente(s). Una(s) queja(s) justificadas fundada(s) - injustificada(s) - infundada(s) 1 une(des) plainte(s) justifiées - injustifiée(s) - fondée(s), non fondée(s). Adv. : (quejarse) enérgicamente / (se plaindre) énergiquement. (quejarse) amargamente /(se plaindre) amèrement. (quejarse) repetidamente, frecuentemente/(se plaindre) continuellement, fréquemment / quejarse de vicio/se plaindre sans raison.

4 - Se plaindre (denunciar/présenter une plainte).

Constr.: a - Max ha denunciado un/el robo (el robo de su radio) (Nhum $+\mathrm{V}+$ $\mathrm{N})$ / Max a présenté une plainte pour vol (pour le vol de sa radio) (Nhum $+\mathrm{V}+-$ pour $\mathrm{N}$ ). $\mathrm{b}-$ Max ha denunciado al cliente por el robo (Nhum $+\mathrm{V}+\mathrm{a}$ Nhum + por $\mathrm{N}) / \mathrm{Max} \mathrm{a}$ présenté une plainte contre le client pour vol (pour le vol de la radio) (Nhum $+\mathrm{V}+$ contre Nhum + pour $\mathrm{N}) \cdot \mathrm{c}-$ Max ha denunciado al cliente por robar $(\mathrm{Nhum}+\mathrm{V}+\mathrm{a}$ Nhum + 
por Vinf.)/Max a présenté une plainte contre le client pour vol (pour le vol de la radio) (Nhum $+\mathrm{V}+$ contre Nhum + pour $\mathrm{N}$ ).

Cooc. lex. : V.: Presentar una denuncia/porter plainte.

3. En guise de conclusion, nous dirons que l'élaboration du dictionnaire dont nous venons de présenter un échantillon aura, à notre avis, des conséquences de type:

- lexicographique: nous montrerons quelles sont les données qui doivent figurer dans un dictionnaire d'encodage ainsi que leur ordonnancement dans un dictionnaire à visée pratique mais s'appuyant en même temps sur des principes linguistiques clairement déterminés et mis en œuvre de manière exhaustive;

- linguistique: en ce qui concerne le contraste des deux langues visées: aucun ouvrage n'offre en parallèle le fonctionnement syntaxique et la cooccurrence lexicale pour deux unités sémantiques équivalentes appartenant à l'espagnol et au français. Cette mise en contraste mènera peut-être, pour certains secteurs, à des constatations intéressantes sur la manière dont chacune des langues visées organise ses sens.

\section{NOTES}

1. Les dictionnaires bilingues copient généralement d'une manière servile les méthodes de la lexicographie monolingue comme l'a montré Wexler (1987) pour un secteur déterminé des lexiques français et anglais «Affix-entries in bilingual dictionaries» in Cahiers de lexicologie, $\mathrm{n}^{\circ}$ 50, 1987-1.

2. M. Snell-Hornby (1986), The Bilingual Dictionary-Victim of its own tradition? in The History of Lexicography, Papers from the Dictionary Centre Seminar at Exeter, March 1986, Ed. R.R.K. Hartmann, Amsterdam/Philadelphia, p. 216.

It seems particularly necessary at the present stage of bilingual lexicography, a renewed spirit of critical inquiry to lay the foundations for new concepts and methods to cope with new and yaried needs.

3. Cf. Gross (a), (b) et (c) et Guillet et La Fauci, 1984.

4. Cf. Zgusta L., 1984 , p. 147 et aussi M. Snell-Homby (1986), The Bilingual Dictionary-Victim of its own tradition? in The History of Lexicography, Papers from the Dictionary Centre Seminar at Exeter, March 1986, Ed. R.R.K., Hartmann, Amsterdam/Philadelphia, p. 216, It does [...] seem that bilingual lexicography has been manoeuvred into a stereotyped pattern of "translation equivalents», a principle which [...] clashes with the notion that cultures and concepts must be viewed in their own terms, demanding in heuristic method of discovery procedures. [...] This means that the bilingual dictionary should [...] not limit language to the status of a mere code consisting of varying labels.

5. Ce qui parâit une exigence minimale mais qui cependant ne se trouve pas toujours respectée : certains dictionnaires fournissent parfois seulement une glose du terme en entree.

6. Notons que, si l'adoption de critères de ce genre - on l'a dit — est à la base de l'organisation de la macrostructure du Dictionnaire du français contemporain déjà nommé, à notre connaissance, aucun dictionnaire espagnol n'a adopté de principes semblables. En outre ces critères - croyons-nous — n'ont jamais été utilisés dans la lexicographie bilingue.

7. Le dernier étant - croyons-nous - le Diccionario Fundamental del Francés, J. Cantera, E. de Vicente, Anaya-Madrid, 1980, qui s'appuie sur la sélection du F.F. (Prologue, p. 1, Están todas las [palabras] del Francés Fundamental en sus dos grados). Ce petit répertoire bilingue ne modifie en rien le système d'équivalences interlinguales de $n$ 'importe quel dictionnaire (bilingue) conventionnel et l'analyse linguistique sous-jacente nécessaire à la rénovation de la lexicographie bilingue en est absente. Nous signalerons cependant que ces auteurs déclarent dans leur prologue que leurs intentions sont de nature essentiellement pédagogique.

8. Ce dernier verbe a déjà fait l'objet d'une présentation dans la communication que nous avons déjà signalée (Lépinette, 1989). Cependant nous avons dans l'actuelle présentation modifié certains points de détail. 


\section{BIBLIOGRAPHIE}

BENSON, M., E. BENSON, R.ILSON (1986), The BBI Combinatory Dictionary of English, Amsterdam, John Benjamins.

DEBYSER, F. (1973), Le lexique des constructions verbales, Multigraphié, Paris, BELC.

DUBOIS, J. (1962), «Recherches lexicographiques: esquisse d'un dictionnaire structural», Études de linguistique appliquée, no 1 .

DUBOIS, J. et al. (1966), Dictionnaire du français contemporain, Paris, Larousse

DUBOIS, J. et C. DUBOIS (1971), Introduction à la lexicographie : le dictionnaire, Paris, Larousse.

CHOUL, J.-C. (1987): «Contrôle de l'équivalence dans les dictionnaires bilingues», in R. Ilson (ed.), : $A$ Spectrum of Lexicography, Amsterdam, John Benjamins, p. 75-90.

ELIA, A. (1984a) : «Étude formelle de différents emplois sémantiques d'un mot, un exemple d'application du lexique-grammaire de l'italien et du français», Cahiers de lexicologie, no 44

ELIA, A. (1984b), «Sur l'unité du mot et la syntaxe comparée des langues romanes», Revue québécoisc de linguistique.

FRADIN, B. et J.-M. MARANDIN (1979), «Autour de la définition, de la lexicographie à la sémantique», Langue française, $\mathrm{n}^{\circ} 43$.

GARCIA-PELAYO, R. GROSS (1987), Diccionario moderno francés-español-español-francés, Paris, Larousse, 1re éd., 1967.

GOUGENHEIM, G. (1958), Dictionnaire fondamental de la langue française, Paris, Didier

GUILLET, A. et N. LA FAUCI (1984), Le lexique grammaire des langues romanes, Amsterdam, John Benjamins.

GROSS, M. (1968), Grammaire transformationnelle du français : syntaxe du verbe, Paris, Larousse.

GROSS, M. (1975), Méthodes en syntaxe, Paris, Hermann.

GROSS, M. (1977), Grammaire transformationnelle du français: syntaxe du nom, Paris, Larousse.

HOUSEHOLDER, F. W, et S. SAPORTA (1968), Problems in Lexicography, Indiana University Bloomington, La Haye-Paris Mouton.

ILSON, R. F. (1987) : A Spectrum of Lexicography, Amsterdam, John Benjamins.

LE GOFFIC, P. et N. COMBE (1975), Les constructions fondamentales du français, Paris, Hachette-Larousse.

LÉPINETTE, B. (1988a), «La définition des émotions en français et en espagnol», Revue québécoise de linguistique, vol. 17, no 2 .

LÉPINETTE, B. (1988b), «Lexicografía bilingüe francés-español y las operaciones de codificación y descodificación», Comunicación presentada en la reunión annual de la Asociación Española de Lingüística Aplicada, (AESLA) Santander, avril 1988.

LÉPINETTE, B. (1989), «Vers un dictionnaire explicatif et combinatoire bilingue», Cahiers de lexicologie (à paraître).

MEL'ČUK, I. et al. (1984), Dictionnaire explicatif et combinatoire du français contemporain, Montréal, Presses de I'Université de Montréal.

REY, A. (1977), Le lexique, images et modèles, Paris, Armand Colin.

REY-DEBOVE, J. (1970), «Le domaine du dictionnaire», Langages, no 19.

REY-DEBOVE, J. (1971), Étude linguistique et sémantique des dictionnaires français contemporains, La HayeParis, Mouton.

VIVES, R. (1985), «Lexique-grammaire et didactique du français langue étrangère», Langue française, no 68 ZGUSTA, L. (1971), Manual of Lexicography, La Haye-Paris, Mouton.

ZGUSTA, L. (1984), «Translational Equivalence in the Bilingual Dictionary» in LEXeter' 83 Proceedings, ed. R.R.K. Hartmann, (Lexicographia. Series Maior) Tübingen, Max Niemeyer, p. 147-154. 